\title{
Selective inactivation of Gram-positive bacteria in vitro and in vivo through metabolic labelling
}

\author{
Tao Feng ${ }^{1 \dagger^{*}}$, Hui $\mathrm{Lu}^{1 \dagger}$, Xiaoting $\mathrm{Ye}^{1 \dagger}$, Chaofan $\mathrm{Nie}^{1}$, Jianhong Zhang ${ }^{1}$, Luofeng $\mathrm{Yu}^{1}$, Haoyu Jin ${ }^{1}$, \\ Peng $\mathrm{Li}^{1^{*}}$ and Wei Huang ${ }^{1,2,3}$
}

\begin{abstract}
Bacterial infections are grave threats to human health, particularly those caused by the most common Grampositive bacteria. The massive administration of broad-spectrum antibiotics to treat various bacterial infections has led to the evolution and spread of drug resistance. As a universal antimicrobial technique unapt to induce drug resistance, photothermal therapy (PTT) is attracting extensive attention in recent years. However, its unspecific killing capability and side effects towards adjacent mammalian cells severely impede the practical applications. Herein, we proposed a metabolic engineering strategy to selectively inactivate Gram-positive bacteria by PTT. A bioorthogonal photothermal agent was prepared by the conjugation of IR-780 iodide and dibenzocyclooctyne (IR780-DBCO). Upon pre-metabolizing with 3azido-D-alanine, Gram-positive bacteria rather than Gramnegative ones, such as Staphylococcus aureus and vancomycinresistant Enterococcus faecalis (VRE), could be specifically tied up by the explosive IR780-DBCO via copper-free click chemistry. Thereafter, they spontaneously detonated under $15 \mathrm{~min}$ near-infrared light irradiation and inactivated nearly $100 \%$ Gram-positive bacteria in vitro. Moreover, superbug VRE-induced infection was significantly inhibited by this approach in a mouse skin wound model. This metabolic labelling-based photothermal ablation strategy specific to Gram-positive microbes would stimulate the development of precise antibacterial candidates for preclinical applications.
\end{abstract}

Keywords: Gram-positive bacteria, selective inactivation, photothermal agent, metabolic labelling, wound infection

\section{INTRODUCTION}

Bacterial infectious diseases are severe challenges to human health, leading to significantly social and medical concerns for a long time $[1,2]$. As a primary category of clinically significant pathogens, Gram-positive bacteria induce serious infections with high morbidity and mortality [3,4], such as sepsis that annually accounts for nearly 1.7 million cases and 270,000 deaths in the United States, as well as 30 million cases and 6 million deaths all around the world $[5,6]$. Generally, bacterial infections are cured by broad-spectrum antibiotics, while their global overuse or abuse has accelerated the evolution and spread of drug resistance [7-9]. In addition, this situation is deteriorated as the production of novel antimicrobial agents is increasingly difficult and costly [10]. As reported by the World Health Organization (WHO), the drug-resistant bacterial infections claim more than 700,000 lives every year, and if current trends continue, the number could rise to 10 million by 2050 with an estimated economic cost of 100 trillion dollars $[11,12]$. Therefore, it is highly desirable to develop effective antimicrobial agents incapable of inducing drug resistance in the post-antibiotic era, especially those specific to Gram-positive bacteria [13].

Recently, photothermal therapy (PTT) has attracted extensive attention for bactericidal applications, on the basis of generating localized hyperthermia to inactivate enzymes or proteins, disrupt membrane permeability, and disorder metabolic signals upon near-infrared (NIR) light irradiation with photothermal agents [14-17]. PTT holds advantageous characteristics of spatiotemporal control, noninvasiveness, and little possibility to induce drug resistance. Up to now, various photothermal agents have been developed to ablate bacteria, including inorganic gold-silver nanocages, copper sulfide, graphene, and black phosphorus, as well as organic IR-780 iodide (IR780), indocyanine green, polydopamine, and polypyrrole [18-22]. However, PTT suffers from the inability to specifically inactivate bacteria and toxic side effects against adjacent mammalian cells, which seriously limit its practical usage.

Metabolic labelling with unnatural precursors is an emerging technique to engineer cells with chemical tags for subsequent modification of complementary molecules via efficient chemistries [23-25], which has been extensively applied in various fields, including cancer labelling and targeting [24], microbiota identification [26-28], and bacterial inactivation [29-31]. A variety of artificial precursors such as chemically modified galactosamine, mannosamine, sialic acid, and fucose are used for cell membrane labelling. Benefitting from the specific components of bacterial cell wall, $D$-alanine and 3-deoxy- $D$-mannooctulosonic acid derivatives are metabolized into the peptidoglycan and lipopolysaccharide, respectively [32,33]. There-

\footnotetext{
${ }^{1}$ Frontiers Science Center for Flexible Electronics (FSCFE), Xi'an Institute of Flexible Electronics (IFE), Xi'an Institute of Biomedical Materials and Engineering (IBME), Ningbo Institute \& Chongqing Technology Innovation Center, Northwestern Polytechnical University (NPU), Xi'an 710072, China

${ }^{2}$ Key Laboratory of Flexible Electronics (KLOFE) \& Institute of Advanced Materials (IAM), Nanjing Tech University (NanjingTech), Nanjing 211816, China

${ }^{3}$ Key Laboratory for Organic Electronics and Information Displays \& Institute of Advanced Materials (IAM), Nanjing University of Posts and Telecommunications, Nanjing 210023, China

$\dagger$ These authors contributed equally to this work.

" Corresponding authors: (emails: iampli@nwpu.edu.cn (Li P); iamtfeng@nwpu.edu.cn (Feng T))
} 
after, functional fluorescent probes and therapeutic molecules are introduced by bioorthogonal reactions, such as click chemistry between azide and alkyne $[31,34]$. Thus, metabolic labelling could be utilized to precisely conjugate photothermal agents on bacteria for selective pathogenic ablation by PPT.

In this contribution, IR780, a lipophilic cationic heptamethine dye with excellent degradability, low cytotoxicity, and superior photothermal conversion efficiency [35-37], was functionalized with dibenzocyclooctyne (DBCO) to prepare a bioorthogonal photothermal agent (IR780-DBCO) for specific killing of Grampositive bacteria in vitro and in vivo (Fig. 1). Upon metabolizing 3 -azido- $D$-alanine $\left(D\right.$-Ala- $\left.\mathrm{N}_{3}\right)$ into bacterial peptidoglycan, IR780-DBCO was selectively labelled on the surface of Grampositive bacteria rather than Gram-negative ones via copper-free click chemistry between azide and DBCO. The specific labelling resulted from the poor permeability of Gram-negative bacterial outer membrane against IR780-DBCO with a molecular weight larger than $650 \mathrm{Da}[31,38]$. Therefore, typical Gram-positive pathogenic bacteria, including Staphylococcus aureus (S. aureus) and vancomycin-resistant Enterococcus faecalis (VRE), were selectively ablated under NIR light irradiation in vitro and in vivo.

\section{EXPERIMENTAL SECTION}

\section{Materials and characterizations}

The experimental details of materials, characterizations, synthesis of IR780-(3-MPA) and IR780-DBCO, photothermal heating curves measurement, bacteria culture and metabolic modification, morphology observation, cytotoxicity and hemolysis assays are described in the Supplementary information.

\section{Bacterial labelling}

One milliliter of $D$-Ala- $\mathrm{N}_{3}$-pretreated or unpretreated bacterial suspension (S. aureus, VRE, Escherichia coli (E. coli), or Pseudomonas aeruginosa (P. aeruginosa)) in a 1.5-mL Eppendorf tube was centrifuged at $2300 \times g$ for $5 \mathrm{~min}$. After the supernatant was discarded, bacteria were resuspended in $200 \mu \mathrm{L}$ of DBCO-
Cy3 solution $\left(20 \mu \mathrm{mol} \mathrm{L}^{-1}\right.$, phosphate buffer saline (PBS)/dimethyl sulfoxide (DMSO) $(v / v)=99 / 1)$ and incubated at room temperature in the dark for $30 \mathrm{~min}$. The labelled bacteria were harvested and washed with PBS for four times. Upon fixing in $1 \mathrm{~mL}$ of $4 \%$ paraformaldehyde at $4^{\circ} \mathrm{C}$ overnight, the bacteria were collected and washed with PBS for four times. Then $1 \mathrm{~mL}$ PBS was added to resuspend the labelled bacteria. The above bacterial suspension $(10 \mu \mathrm{L})$ was transferred into a confocal dish and covered by a clean cover glass for imaging. The images were recorded by an inverted fluorescence microscope with excitation wavelength of $530 \mathrm{~nm}$ and emission wavelength of $593 \mathrm{~nm}$, and a structured illumination microscope (SIM) with excitation wavelength of $561 \mathrm{~nm}$ and emission wavelength of $600-660 \mathrm{~nm}$.

\section{In vitro selective antibacterial assays}

The antibacterial activity of IR780-DBCO towards S. aureus, VRE, E. coli, or $P$. aeruginosa was evaluated by a standard agar plating method. First, $1 \mathrm{~mL}$ of the pretreated bacteria in a $1.5-\mathrm{mL}$ Eppendorf tube was centrifuged at $2300 \times g$ for $5 \mathrm{~min}$ to discard the supernatant. Second, the bacteria were suspended in $200 \mu \mathrm{L}$ of IR780-DBCO solutions with various concentrations $(0,2.5,5$, or $\left.10 \mu \mathrm{mol} \mathrm{L}{ }^{-1}, \mathrm{PBS} / \mathrm{DMSO}(v / v)=99 / 1\right)$, and incubated at room temperature in the dark for $30 \mathrm{~min}$. Then the labelled bacteria were harvested and washed with PBS for three times, and subsequently resuspended in $400 \mu \mathrm{L}$ PBS. For the antibacterial activity of IR780-DBCO under NIR light irradiation, $200 \mu \mathrm{L}$ of the above-prepared bacterial suspension was transferred to a sterile disposable petri dish and irradiated by $808-\mathrm{nm}$ NIR laser $\left(1.0 \mathrm{~W} \mathrm{~cm}^{-2}\right)$ for $15 \mathrm{~min}$. For the antibacterial activity of IR780DBCO in the dark, $200 \mu \mathrm{L}$ of the above-prepared bacterial suspension was placed in the dark for $15 \mathrm{~min}$ under the same conditions. Afterwards, $1.8 \mathrm{~mL}$ PBS was added to the petri dish, followed by sonication for $3 \mathrm{~min}$ to disperse the bacteria. One hundred microliter of the suspension was taken for 10-fold gradient dilution. Ten microliter of the diluted bacterial solution was inoculated in an agar plate, and the colony forming units (CFUs) were counted after incubation at $37^{\circ} \mathrm{C}$ overnight. In addition, $50 \mu \mathrm{L}$ of the suspension diluted by 100 folds was spread

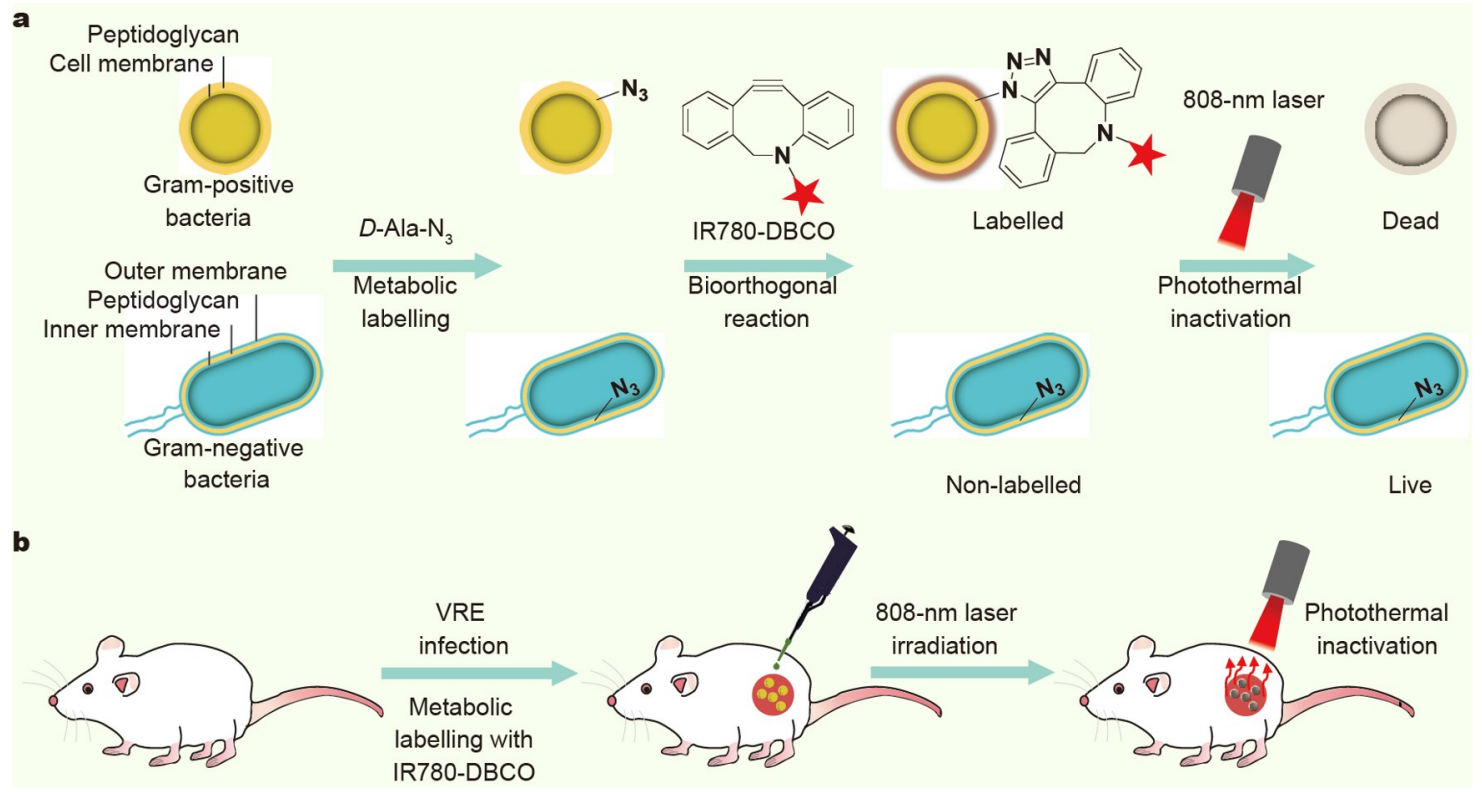

Figure 1 Schematic illustration of IR780-DBCO for selective photothermal inactivation of Gram-positive bacteria (a) in vitro and (b) in vivo. 
on the agar plate. The bacterial viability $(\%)=\mathrm{CFU}_{\text {experiment }} /$ $\mathrm{CFU}_{\text {control }} \times 100 \%$, where $\mathrm{CFU}_{\text {experiment }}$ and $\mathrm{CFU}_{\text {control }}$ were the average CFU of the experimental group with IR780-DBCO treatment and the control group with PBS treatment, respectively. All experiments were repeated for three times. Using the similar method, the antibacterial activity of IR780-DBCO (0 and $\left.5 \mu \mathrm{mol} \mathrm{L}{ }^{-1}, \mathrm{PBS} / \mathrm{DMSO}(v / v)=99 / 1\right)$ towards $S$. aureus, VRE, $E$. coli, or $P$. aeruginosa without $D$-Ala- $\mathrm{N}_{3}$ pretreatment was evaluated in the dark or under NIR laser irradiation. In addition, the killing effect of IR780, IR780-(3-MPA), or IR780-DBCO (0 and $5 \mu \mathrm{mol} \mathrm{L}{ }^{-1}$, PBS/DMSO $\left.(v / v)=99 / 1\right)$ against $D$-Ala- $\mathrm{N}_{3}$-pretreated or unpretreated VRE was measured in the dark or under NIR light illumination.

\section{In vivo anti-infective assay}

The in vivo anti-infective ability of IR780-DBCO was assessed in a mouse model with VRE-infected wound. All procedures were approved by the Animal Ethical Committee of Northwestern Polytechnical University. Female BALB/c mice (6-8 weeks) were purchased from Beijing Vital River Laboratory Animal Technology Co., Ltd.

Twelve BALB/c mice were randomly divided into four groups: (1) control group only with bacterial infection, (2) bacteriainfected group under 808-nm NIR laser irradiation, (3) bacteriainfected group treated with IR780-DBCO, and (4) bacteriainfected group treated with IR780-DBCO and 808-nm NIR laser irradiation. The mice were anesthetized using isoflurane and then the open excision wounds were created on the dorsal skin using an 8 -mm diameter biopsy punch. The bacteria-infected skin wound models were established by inoculating $50 \mu \mathrm{L}$ of the pretreated VRE suspension $\left(10^{4} \mathrm{CFU}\right)$ on the wound area. For the groups with IR780-DBCO treatment, a total of $50 \mu \mathrm{L}$ of the pretreated VRE suspension and IR780-DBCO (the final concentration was $20 \mu \mathrm{mol} \mathrm{L}^{-1}$ ) were added onto each wound and kept for $30 \mathrm{~min}$. Afterwards, the wounds were irradiated by $808-\mathrm{nm}$ NIR laser $\left(1.0 \mathrm{~W} \mathrm{~cm}^{-2}\right)$ for $15 \mathrm{~min}$ or kept in the dark. The treatments by IR780-DBCO and with or without $808-\mathrm{nm}$ NIR laser irradiation were performed for total three times. The wound areas were photographed using an 8 -mm rubber ring as reference every day. After four days, the mice were sacrificed and the entire wounds with the adjacent skin tissues were harvested and placed in $1 \mathrm{~mL}$ PBS followed by sonication for $15 \mathrm{~min}$. The CFUs of bacteria in infectious tissues were evaluated by the standard agar plating method. The collected wound tissues were fixed in $4 \%$ paraformaldehyde at $4^{\circ} \mathrm{C}$, embedded in paraffin, and cut into slices for histopathological hematoxylin-eosin (H\&E) staining.

\section{RESULTS AND DISCUSSION}

\section{Synthesis and characterization of IR780-DBCO}

To enable photothermal property and the reactivity with azide group, IR780 was firstly conjugated with 3-mercaptopropionic acid (3-MPA) through nucleophilic substitution reaction between sulfhydryl and chlorine groups to produce IR780-(3MPA) in a $64 \%$ yield, and subsequently reacted with DBCOamine $\left(\mathrm{DBCO}-\mathrm{NH}_{2}\right)$ through amide condensation reaction to prepare IR780-DBCO in a $43 \%$ yield (Fig. S1 and Fig. 2a). As confirmed by nuclear magnetic resonance and mass spectra, IR780-DBCO was successfully synthesized. The optical characteristic of IR780-DBCO was displayed in Fig. 2b, with the absorption lying in the broad ultra violet-visible-NIR (UV-VisNIR) range from 200 to $900 \mathrm{~nm}$ and the maximal peak located at $785 \mathrm{~nm}$. Moreover, the temperature evolution curves of IR780-

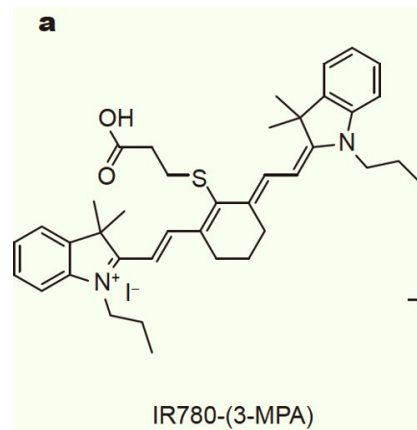

b

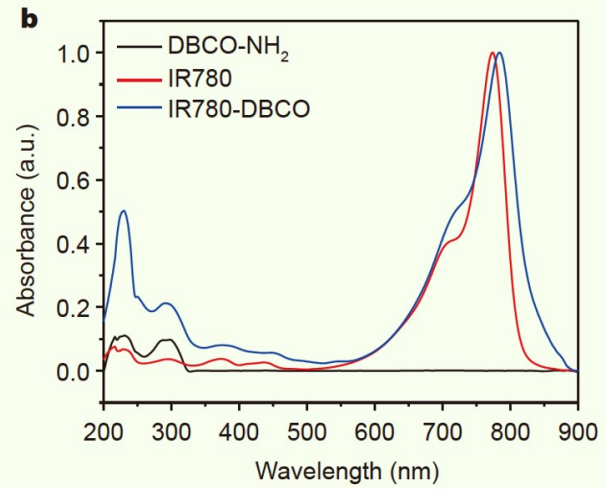

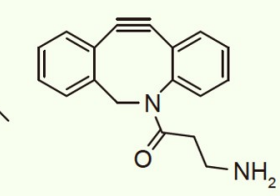
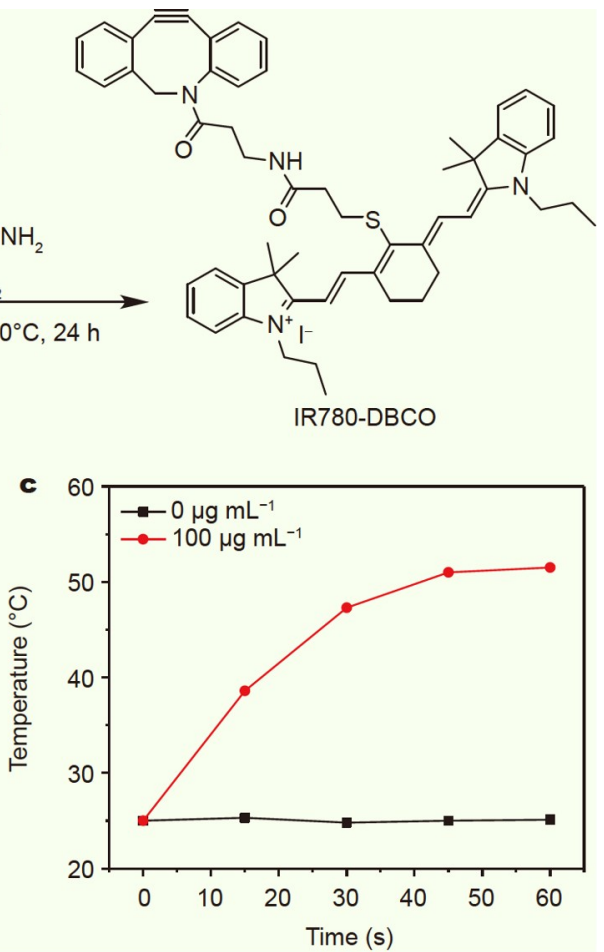

Figure 2 (a) Synthetic route of IR780-DBCO. (b) UV-Vis-NIR absorption spectra of DBCO-NH 2 , IR780, and IR780-DBCO in DMSO/PBS mixtures $(v / v=$ 1/99). DMF: N,N-dimethylformamide; EDC: 1-(3-dimethylaminopropyl)-3-ethylcarbodiimide hydrochloride; HOAT: 1-hydroxy-7-azabenzotriazole.

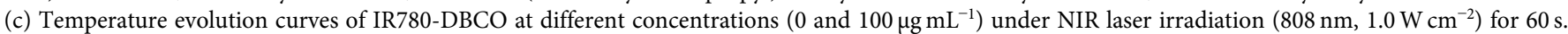


DBCO were measured under 808-nm NIR laser irradiation with a power density of $1.0 \mathrm{~W} \mathrm{~cm}^{-2}$ (Fig. 2c). At a concentration of $100 \mu \mathrm{g} \mathrm{mL}^{-1}$, the temperature increased by $26.5^{\circ} \mathrm{C}$ upon irradiation for $60 \mathrm{~s}$, while it had negligible change at $0 \mu \mathrm{g} \mathrm{mL}^{-1}$ under the same condition. Upon IR780-DBCO reacting with $D-A l a-N_{3}$ through click chemistry, there were no significant differences for the UV-Vis-NIR spectrum and temperature evolution curve when compared with those for IR780-DBCO alone (Fig. S2). These results revealed that IR780-DBCO possessed an outstanding photothermal effect, which was not influenced by the click reaction, and held great potential for eliminating bacteria.

\section{Selective bacterial labelling}

In order to evaluate the specific labelling of Gram-positive bacteria by metabolic engineering, two Gram-positive bacteria (S. aureus and VRE) and two Gram-negative bacteria (E. coli and $P$. aeruginosa) were pretreated with $D$-Ala- $\mathrm{N}_{3}$ to integrate azide groups into the peptidoglycan of bacteria, followed by bioorthogonal clicking with fluorescent DBCO-Cy3, a commercial dye containing alkyne groups. As shown in Fig. 3, Gram-positive $S$. aureus and VRE were stained with strong red fluorescence, while Gram-negative E. coli and $P$. aeruginosa presented almost no fluorescence. Without pretreatment with $D$-Ala- $\mathrm{N}_{3}$, all types of bacteria showed negligible fluorescence, indicating the efficient click reaction between $\mathrm{DBCO}-\mathrm{Cy} 3$ and metabolic precursor (Fig. S3). Most importantly, to demonstrate the exact region of labelled $\mathrm{DBCO}-\mathrm{Cy} 3$, super resolution imaging of bacteria was performed by SIM. The obvious fluorescence was

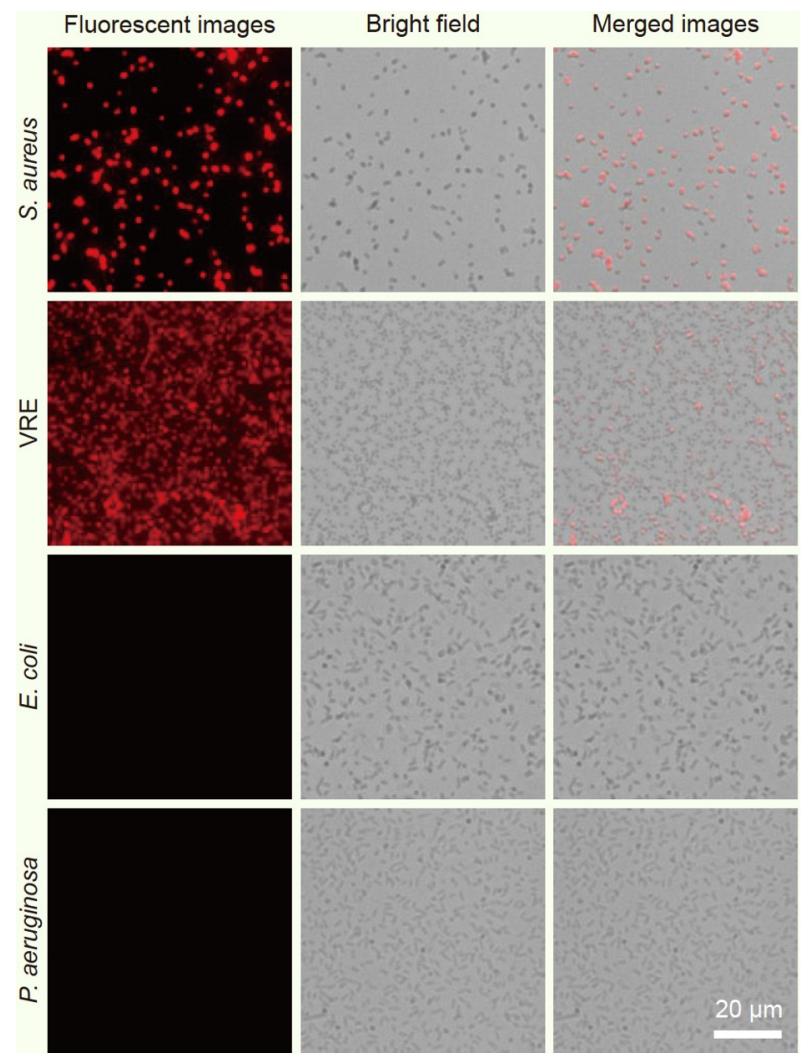

Figure 3 Fluorescent images of Gram-positive bacteria ( $S$. aureus and VRE) and Gram-negative bacteria (E. coli and P. aeruginosa) upon incubation with $D$-Ala- $\mathrm{N}_{3}$ and DBCO-Cy3. The fluorescent channel was recorded with excitation wavelength of $530 \mathrm{~nm}$ and emission wavelength of $593 \mathrm{~nm}$. only observed around the periphery of Gram-positive bacteria (S. aureus and VRE), indicating the preferential labelling of their cell walls (Fig. 4). The underlying mechanism for selective imaging of Gram-positive bacteria could be explained as follows: $D$-Ala- $\mathrm{N}_{3}$ was able to metabolize into peptidoglycan of bacterial cell wall to endow azide groups owing to the presence of $D$ alanine motif [32]. The outer membrane of Gram-negative bacteria is less permeable to molecules with molecular weight larger than $650 \mathrm{Da}$ [31], and thus DBCO-Cy3 with molecular weight of $1186 \mathrm{Da}$ was specifically labelled on Gram-positive bacteria through copper-free click chemistry between azide and DBCO groups. Moreover, while traditional culture-based methods for bacterial identification usually require several days, this metabolic labelling approach is much faster and effective.

\section{Photothermal antibacterial study}

Because of the similar chemical structure to DBCO-Cy3, IR780DBCO with a molecular weight of $994 \mathrm{Da}$ could be specifically loaded on the cell envelope of $D$-Ala- $\mathrm{N}_{3}$-pretreated Grampositive bacteria, which is advantageous for photothermal inactivation of pathogens. We then assessed the selective antibacterial capability of IR780-DBCO using the standard plate counting method [20]. Gram-positive bacteria (S. aureus and VRE) and Gram-negative ones (E. coli and P. aeruginosa) were metabolized with $D$-Ala- $\mathrm{N}_{3}$ and incubated with IR780-DBCO at different concentrations, followed by treatment in dark or under 808-nm NIR laser irradiation at a power density of $1.0 \mathrm{~W} \mathrm{~cm}^{-2}$ for $15 \mathrm{~min}$. For Gram-positive bacteria, IR780-DBCO showed concentration-dependent inhibition under NIR light irradiation, with the killing efficiency of $99.7 \%$ for S. aureus or $100 \%$ for VRE, and few bacterial colonies formed on the agar plates at a concentration of $10 \mu \mathrm{mol} \mathrm{L}^{-1}$ (Fig. 5a-d). Nevertheless, NIR light irradiation or IR780-DBCO alone presented negligible killing effect on Gram-positive bacteria, which grew smoothly on the agar plates. For Gram-negative bacteria, the killing effect was insignificant upon treatment with NIR light irradiation, IR780DBCO, or IR780-DBCO plus NIR light irradiation, resulting in obvious colony formation similar to the control group (Fig. 5eh). Without $D$-Ala-N $\mathrm{N}_{3}$ pretreatment, the viability of all types of bacteria was much higher upon incubation with equivalent IR780-DBCO or plus NIR light irradiation (Fig. S4). Moreover,
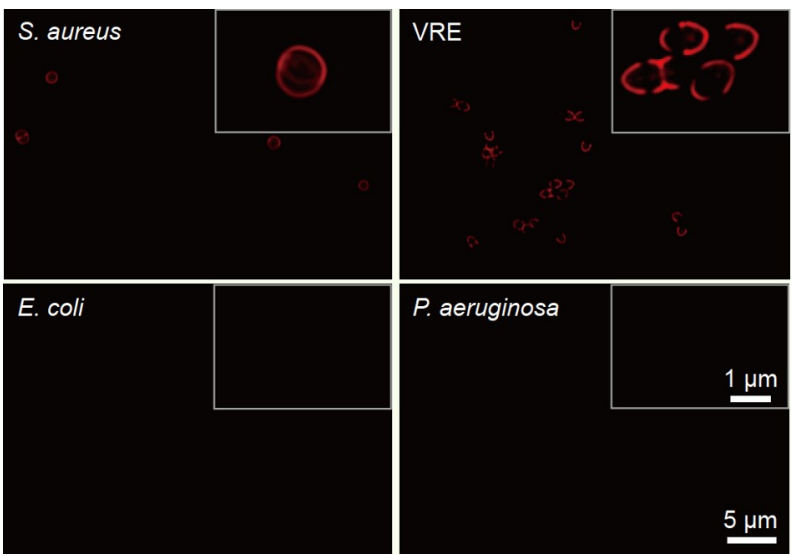

Figure 4 SIM images of Gram-positive bacteria (S. aureus and VRE) and Gram-negative bacteria (E. coli and $P$. aeruginosa) upon incubation with $D$ Ala- $\mathrm{N}_{3}$ and DBCO-Cy3. The fluorescence was measured with excitation wavelength of $561 \mathrm{~nm}$ and emission wavelength of $600-660 \mathrm{~nm}$. 
for Gram-positive VRE, the killing effect of IR780 and IR780-(3MPA) without or with $D$-Ala- $\mathrm{N}_{3}$ pretreatment was much lower than that incubated with $D$-Ala- $\mathrm{N}_{3}$ and equivalent IR780-DBCO under NIR light illumination (Fig. S5). These results suggested that Gram-positive bacteria rather than Gram-negative ones could be selectively and effectively eliminated by PTT through metabolic labelling of IR780-DBCO on the cell wall.

Moreover, in order to elucidate the antibacterial mechanism, scanning electron microscopy (SEM) was utilized to observe the morphological changes of bacteria after different treatments. Upon metabolic labelling with IR780-DBCO and irradiation with NIR light, the cell envelopes of Gram-positive bacteria were seriously destroyed, with obvious deformation and collapse for $S$. aureus and obvious wrinkle for VRE, as indicated by the white arrows in Fig. 6 and Fig. S6. However, the morphology of Grampositive bacteria treated with NIR light irradiation or IR780DBCO or Gram-negative bacteria with any treatment had insignificant change, displaying intact cell wall and relatively
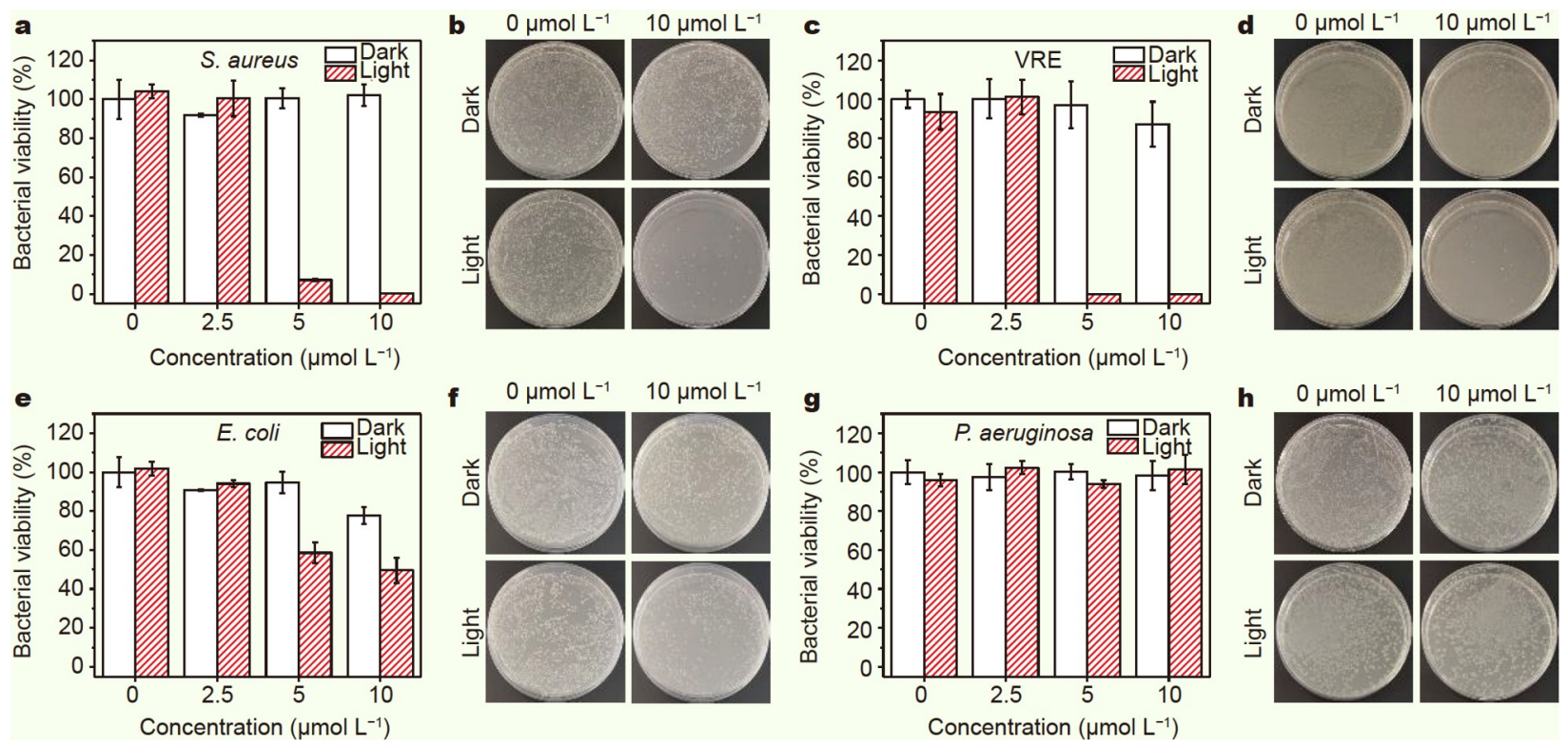

Figure 5 (a, c, e, g) Bacterial viability and (b, d, f, h) photographs of the corresponding colonies of $D$-Ala-N $\mathrm{N}_{3}$-pretreated Gram-positive bacteria, $(\mathrm{a}, \mathrm{b})$ S. aureus and (c, d) VRE, and Gram-negative bacteria, (e, f) E. coli and (g, h) P. aeruginosa, upon incubation with IR780-DBCO at various concentrations and irradiation with/without 808-nm laser at a power density of $1.0 \mathrm{~W} \mathrm{~cm}^{-2}$ for $15 \mathrm{~min}$. Error bars: $\mathrm{mean} \pm \mathrm{SD}(n=3)$.
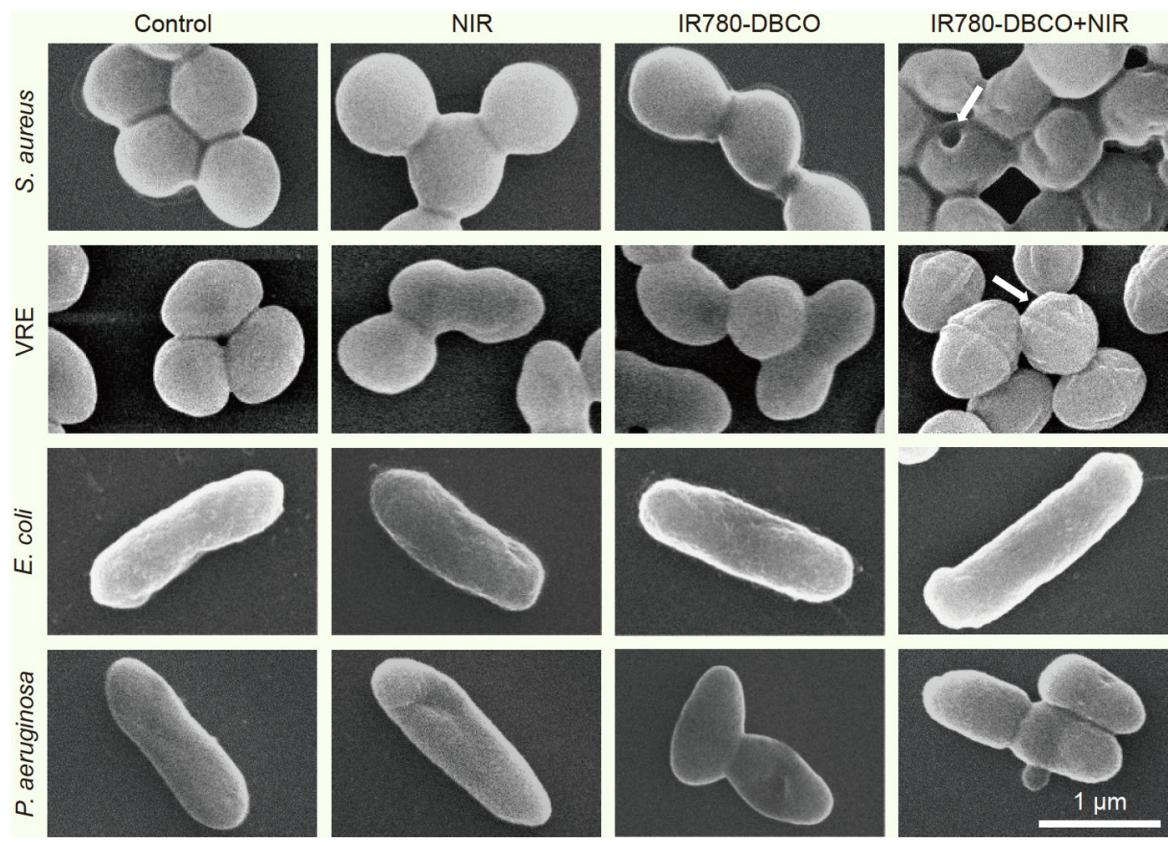

Figure 6 SEM images of $D$-Ala- $\mathrm{N}_{3}$-pretreated Gram-positive bacteria ( $S$. aureus and VRE) and Gram-negative bacteria (E. coli and $P$. aeruginosa) upon incubation with IR780-DBCO of $10 \mu \mathrm{mol} \mathrm{L}^{-1}$ and irradiation with/without 808-nm laser at a power density of $1.0 \mathrm{~W} \mathrm{~cm}{ }^{-2}$ for $15 \mathrm{~min}$. 
smooth surface. These results clearly proved that IR780-DBCO conjugated on the bacterial surface could damage the integrity of bacterial cell walls through PTT [36,39], inducing the selective elimination of Gram-positive bacteria.

\section{In vitro biocompatibility study}

To evaluate the potential of IR780-DBCO as a practical antimicrobial agent, the cytotoxicity of IR780-DBCO against mouse embryonic fibroblast cells (NIH3T3) was determined [40]. As shown by alamarBlue assay, IR780-DBCO exhibited negligible cytotoxicity even at a concentration of $10 \mu \mathrm{mol} \mathrm{L}^{-1}$ (Fig. 7a). Moreover, on the basis of live/dead staining results, NIH3T3 cells incubated with IR780-DBCO were almost alive with analogous morphology to the control cells (Fig. 7b). In addition, blood compatibility of IR780-DBCO was assessed [41]. After incubating rabbit red blood cells (RBCs) with $D$-Ala- $\mathrm{N}_{3}$ and different concentrations of IR780-DBCO, colorless supernatant similar to normal saline group was observed with the hemolysis ratios lower than 1\% (Fig. 7c and Fig. S7). Nevertheless, the supernatant of RBCs treated with hemolytic $0.1 \%$ Triton X-100 showed a red color due to the rupture of cell membrane of RBCs and the release of a large amount of hemoglobin. These results indicated that IR780-DBCO had outstanding biocompatibility towards mammalian cells.

\section{In vivo anti-infective assay}

To further demonstrate the bactericidal efficacy of IR780-DBCO in vivo, we established an animal model with azide-labelled VRE-infected wounds on the back skin of BALB/c mice. Upon different treatments with NIR light irradiation, IR780-DBCO, or IR780-DBCO plus NIR light irradiation, the photographs of wounds were recorded at varied time points. As depicted in Fig. 8a, all the VRE-infected wounds had a certain degree of suppuration on day 2. Moreover, compared with the groups with no treatment or treated with only NIR light irradiation or IR780DBCO, the suppuration in the group treated with IR780-DBCO plus NIR light irradiation was obviously diminished on day 4, proving that IR780-DBCO was capable of inhibiting the bacterial infection at the wound site through PTT. Meanwhile, VRE in the homogenized wound tissues on day 4 was quantified to evaluate the anti-infective effect of IR780-DBCO. The number of bacteria in wound tissues for the control group, NIR light irradiation group, or IR780-DBCO group was $10.87 \times 10^{6}, 8.97 \times 10^{6}$, or $6.30 \times 10^{6} \mathrm{CFU} \mathrm{g}^{-1}$, respectively, which was consistent with the result of a large amount of bacterial colonies presented on the agar plate (Fig. 8b, c). However, the bacterial number detected in IR780-DBCO plus NIR light irradiation treated group reduced by three orders of magnitude, with only $2.76 \times 10^{3} \mathrm{CFU} \mathrm{g}^{-1}$, which indicated the excellent photothermal bactericidal performance of IR780-DBCO. In addition, the wound tissues of mice were collected on day 4 and stained with $\mathrm{H} \& \mathrm{E}$ to investigate the anti-infective ability of IR780-DBCO towards VRE. Generally, infected tissues can generate large numbers of neutrophils, which are stained blue by $H \& E$ staining [42]. As revealed in Fig. 8d, VRE-infected wounds treated with IR780-DBCO plus NIR light irradiation showed much fewer neutrophils than the other groups, implying that IR780-DBCO under NIR laser illumination could significantly eliminate Gram-positive VRE
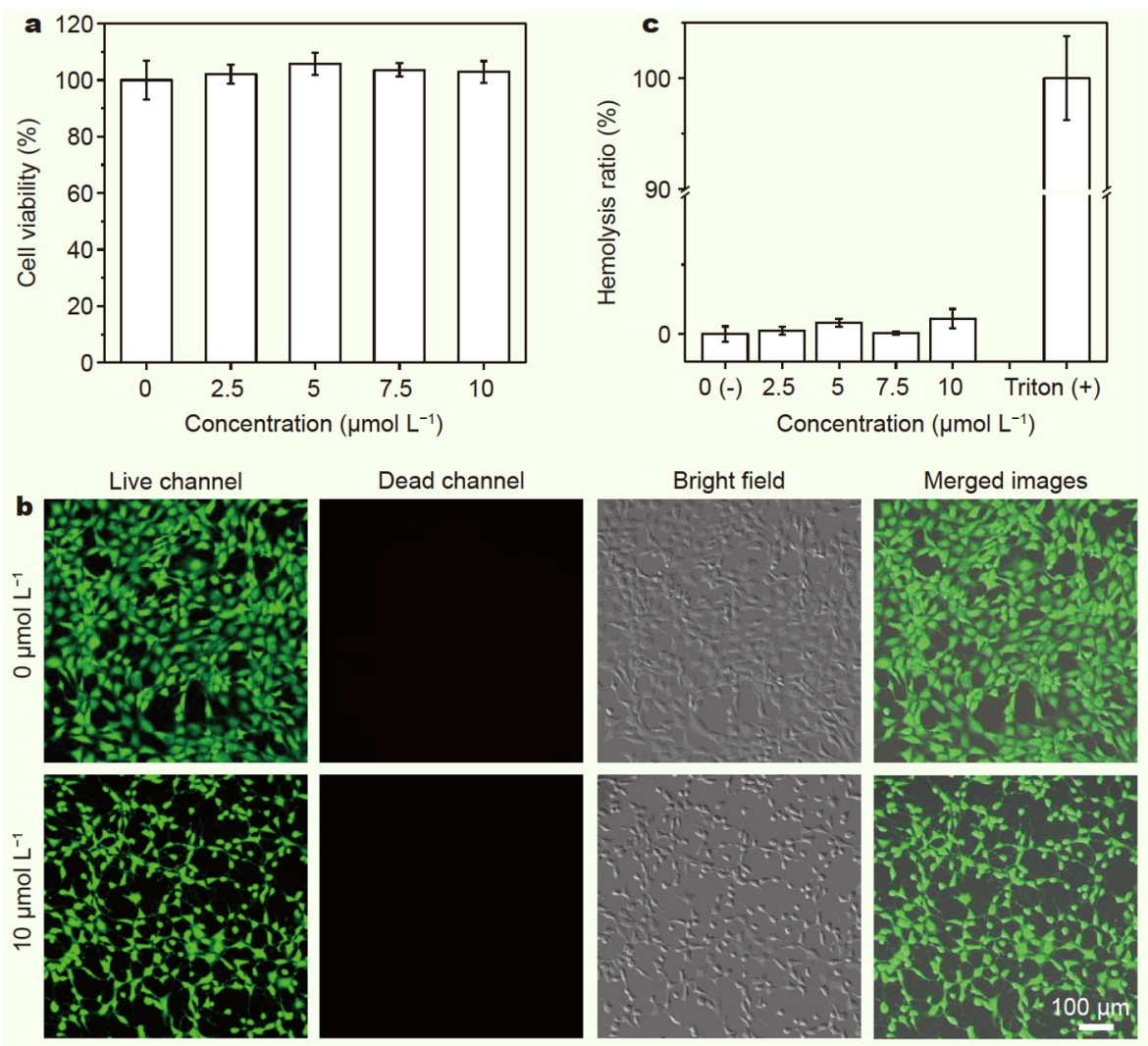

Figure 7 (a) Cell viability and (b) live/dead staining of NIH3T3 cells cultured with D-Ala-N 3 and IR780-DBCO at different concentrations. (c) Hemolysis ratios of RBCs treated with $D$-Ala- $\mathrm{N}_{3}$ and IR780-DBCO at different concentrations, $0.1 \%$ Triton X-100 (positive control), and normal saline (negative control). Error bars: mean $\pm \mathrm{SD}(n=3)$. 

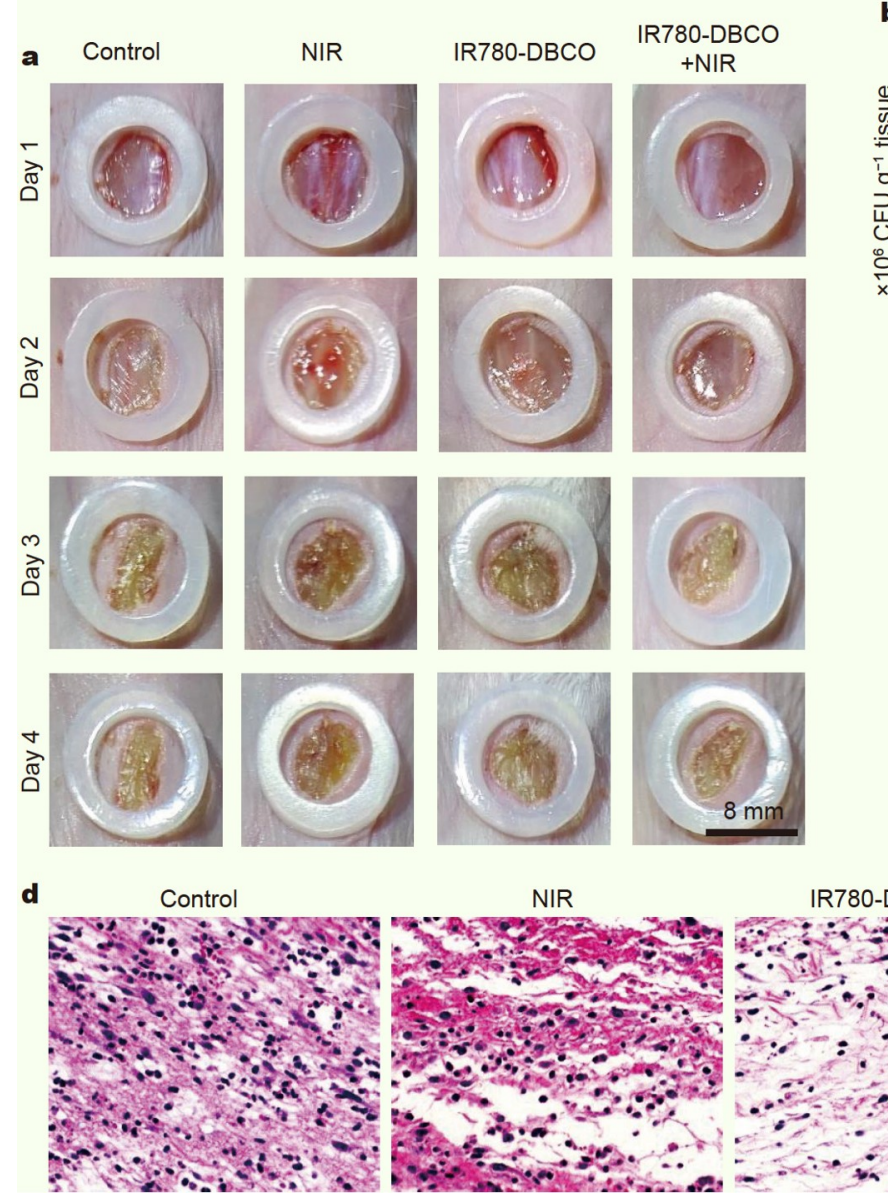

IR780-DBCO

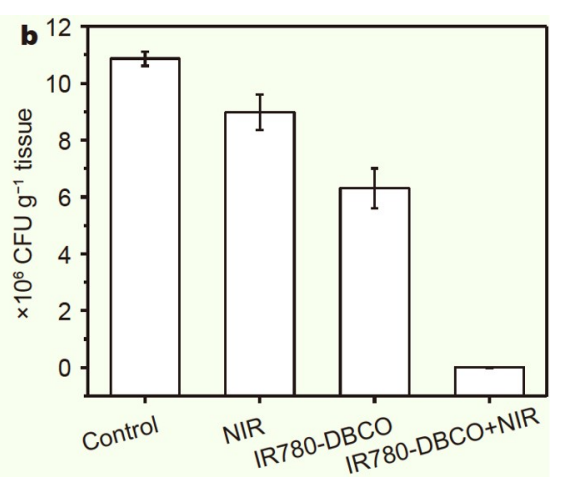

c

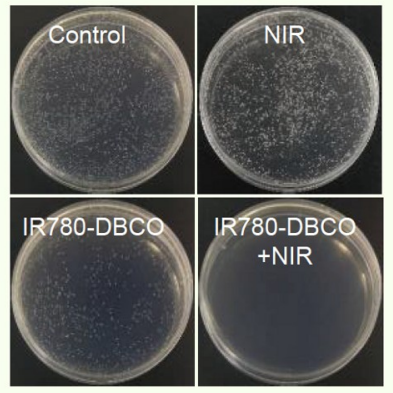

IR780-DBCO+NIR

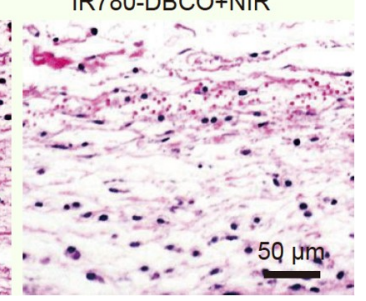

Figure 8 (a) Photographs of VRE-infected wounds of mice with different treatments (Control, NIR light irradiation, IR780-DBCO, or IR780-DBCO plus NIR light irradiation) on days 1, 2, 3, and 4. (b) Quantitative analysis and (c) photographs of bacterial colonies from wound tissues of different treatment groups $(n=3)$. (d) H\&E-stained wound tissues after different treatments for four days.

and effectively suppress bacterial infection at the wounds.

\section{CONCLUSIONS}

In summary, Gram-positive bacteria were selectively inactivated by a bioorthogonal photothermal agent (IR780-DBCO) via metabolic engineering. Followed by pretreating bacteria with metabolic precursor $D$-Ala- $\mathrm{N}_{3}$, the bioorthogonal IR780-DBCO could be specifically conjugated on the surface of Gram-positive bacteria instead of Gram-negative ones through click reaction. Benefitting from the outstanding photothermal conversion performance and excellent biocompatibility of IR780-DBCO, precise inactivation of Gram-positive bacteria, such as $S$. aureus and superbug VRE, was demonstrated under NIR light irradiation. Most importantly, in vivo anti-infective capability was successfully verified in a VRE-infected mouse skin wound model. As compared with the reported nanoplatforms [43-45] or electrostatic and hydrophobic interaction-based strategies [46-48] for selective bacterial imaging and elimination, our method is advantageous in the stable covalent conjugation of photothermal agent on the cell wall with short distance for enhanced and spatiotemporally controlled PTT, as well as the defined or tunable chemical structures and the easy diffusion to infectious sites of small-molecule reagents. This study not only provides valuable practice for designing bioorthogonal photo- thermal molecules and one-step metabolic labelling phototheranostic agents to specifically sterilize Gram-positive bacteria, but also promotes the development of next-generation antimicrobial agents in the post-antibiotic era.

Received 3 April 2021; accepted 10 June 2021; published online 18 August 2021

1 Casadevall A, Pirofski LA. Microbiology: Ditch the term pathogen. Nature, 2014, 516: 165-166

2 Rohr JR, Barrett CB, Civitello DJ, et al. Emerging human infectious diseases and the links to global food production. Nat Sustain, 2019, 2: $445-456$

3 Menichetti F. Current and emerging serious Gram-positive infections. Clin Microbiol Infect, 2005, 11: 22-28

4 Chung HJ, Reiner T, Budin G, et al. Ubiquitous detection of Grampositive bacteria with bioorthogonal magnetofluorescent nanoparticles. ACS Nano, 2011, 5: 8834-8841

5 Rhee C, Dantes R, Epstein L, et al. Incidence and trends of sepsis in US hospitals using clinical vs claims data, 2009-2014. J Am Med Assoc, 2017, 318: 1241-1249

6 Fleischmann C, Scherag A, Adhikari NKJ, et al. Assessment of global incidence and mortality of hospital-treated sepsis. Current estimates and limitations. Am J Respir Crit Care Med, 2016, 193: 259-272

7 Kollef MH. Broad-spectrum antimicrobials and the treatment of serious bacterial infections: Getting it right up front. Clin Infect Dis, 2008, 47: 


\section{S3-S13}

8 Chellat MF, Raguž L, Riedl R. Targeting antibiotic resistance. Angew Chem Int Ed, 2016, 55: 6600-6626

9 Wang $\mathrm{S}, \mathrm{Xu} \mathrm{M}$, Huang $\mathrm{K}$, et al. Biocompatible metal-free organic phosphorescent nanoparticles for efficiently multidrug-resistant bacteria eradication. Sci China Mater, 2020, 63: 316-324

10 Levin-Reisman I, Ronin I, Gefen O, et al. Antibiotic tolerance facilitates the evolution of resistance. Science, 2017, 355: 826-830

11 Ma YX, Wang CY, Li YY, et al. Considerations and caveats in combating ESKAPE pathogens against nosocomial infections. Adv Sci, 2020, 7: 1901872

12 Yu F, Chen C, Yang G, et al. An acid-triggered porphyrin-based block copolymer for enhanced photodynamic antibacterial efficacy. Sci China Chem, 2021, 64: 459-466

13 Ye X, Feng T, Li L, et al. Theranostic platforms for specific discrimination and selective killing of bacteria. Acta Biomater, 2021, 125: $29-40$

14 Ray PC, Khan SA, Singh AK, et al. Nanomaterials for targeted detection and photothermal killing of bacteria. Chem Soc Rev, 2012, 41: 31933209

15 Hsiao CW, Chen HL, Liao ZX, et al. Effective photothermal killing of pathogenic bacteria by using spatially tunable colloidal gels with nanolocalized heating sources. Adv Funct Mater, 2015, 25: 721-728

16 Korupalli C, Huang CC, Lin WC, et al. Acidity-triggered charge-convertible nanoparticles that can cause bacterium-specific aggregation in situ to enhance photothermal ablation of focal infection. Biomaterials, 2017, 116: 1-9

17 Chen Q, He S, Zhang F, et al. A versatile Pt-Ce6 nanoplatform as catalase nanozyme and NIR-II photothermal agent for enhanced PDT/ PTT tumor therapy. Sci China Mater, 2021, 64: 510-530

$18 \mathrm{Xu}$ JW, Yao K, Xu ZK. Nanomaterials with a photothermal effect for antibacterial activities: An overview. Nanoscale, 2019, 11: 8680-8691

19 Zhao Y, Dai X, Wei X, et al. Near-infrared light-activated thermosensitive liposomes as efficient agents for photothermal and antibiotic synergistic therapy of bacterial biofilm. ACS Appl Mater Interfaces, 2018, 10: 14426-14437

20 Lei W, Ren K, Chen T, et al. Polydopamine nanocoating for effective photothermal killing of bacteria and fungus upon near-infrared irradiation. Adv Mater Interfaces, 2016, 3: 1600767

21 Wang $\mathrm{H}$, Li X, Tse BWC, et al. Indocyanine green-incorporating nanoparticles for cancer theranostics. Theranostics, 2018, 8: 1227-1242

22 Cai Y, Si W, Huang W, et al. Organic dye based nanoparticles for cancer phototheranostics. Small, 2018, 14: 1704247

23 Wang $\mathrm{H}$, Tang $\mathrm{L}$, Liu $\mathrm{Y}$, et al. In vivo targeting of metabolically labeled cancers with ultra-small silica nanoconjugates. Theranostics, 2016, 6: 1467-1476

24 Yuan Y, Xu S, Cheng X, et al. Bioorthogonal turn-on probe based on aggregation-induced emission characteristics for cancer cell imaging and ablation. Angew Chem Int Ed, 2016, 55: 6457-6461

25 Sun T, Yu SH, Zhao P, et al. One-step selective exoenzymatic labeling (SEEL) strategy for the biotinylation and identification of glycoproteins of living cells. J Am Chem Soc, 2016, 138: 11575-11582

26 Wang W, Lin L, Du Y, et al. Assessing the viability of transplanted gut microbiota by sequential tagging with $\mathrm{D}$-amino acid-based metabolic probes. Nat Commun, 2019, 10: 1317

27 Wang W, Yang Q, Du Y, et al. Metabolic labeling of peptidoglycan with NIR-II dye enables in vivo imaging of gut microbiota. Angew Chem Int Ed, 2020, 59: 2628-2633

28 Hudak JE, Alvarez D, Skelly A, et al. Illuminating vital surface molecules of symbionts in health and disease. Nat Microbiol, 2017, 2: 1-8

29 Mao D, Hu F, Kenry F, et al. Metal-organic-framework-assisted in vivo bacterial metabolic labeling and precise antibacterial therapy. Adv Mater, 2018, 30: 1706831

30 Hu F, Qi G, Kenry G, et al. Visualization and in situ ablation of intracellular bacterial pathogens through metabolic labeling. Angew Chem Int Ed, 2020, 59: 9288-9292

31 Wu M, Qi G, Liu X, et al. Bio-orthogonal AIEgen for specific discrimination and elimination of bacterial pathogens via metabolic en- gineering. Chem Mater, 2020, 32: 858-865

32 Liang H, DeMeester KE, Hou CW, et al. Metabolic labelling of the carbohydrate core in bacterial peptidoglycan and its applications. Nat Commun, 2017, 8: 15015

33 Dumont A, Malleron A, Awwad M, et al. Click-mediated labeling of bacterial membranes through metabolic modification of the lipopolysaccharide inner core. Angew Chem Int Ed, 2012, 51: 3143-3146

34 Shieh P, Sloan Siegrist M, Cullen AJ, et al. Imaging bacterial peptidoglycan with near-infrared fluorogenic azide probes. Proc Natl Acad Sci USA, 2014, 111: 5456-5461

35 Yan F, Duan W, Li Y, et al. NIR-laser-controlled drug release from DOX/IR-780-loaded temperature-sensitive-liposomes for chemophotothermal synergistic tumor therapy. Theranostics, 2016, 6: 23372351

36 Qing G, Zhao X, Gong N, et al. Thermo-responsive triple-function nanotransporter for efficient chemo-photothermal therapy of multidrug-resistant bacterial infection. Nat Commun, 2019, 10: 4336

37 Kuang Y, Zhang K, Cao Y, et al. Hydrophobic IR-780 dye encapsulated in cRGD-conjugated solid lipid nanoparticles for NIR imaging-guided photothermal therapy. ACS Appl Mater Interfaces, 2017, 9: 1221712226

38 Kuru E, Hughes HV, Brown PJ, et al. In situ probing of newly synthesized peptidoglycan in live bacteria with fluorescent $D$-amino acids. Angew Chem Int Ed, 2012, 51: 12519-12523

39 Wu F, Zheng H, Wang W, et al. Rapid eradication of antibiotic-resistant bacteria and biofilms by MXene and near-infrared light through photothermal ablation. Sci China Mater, 2021, 64: 748-758

40 Zhi Z, Su Y, Xi Y, et al. Dual-functional polyethylene glycol- $b$-polyhexanide surface coating with in vitro and in vivo antimicrobial and antifouling activities. ACS Appl Mater Interfaces, 2017, 9: 10383-10397

41 Yang C, Hu F, Zhang X, et al. Combating bacterial infection by in situ self-assembly of AIEgen-peptide conjugate. Biomaterials, 2020, 244: 119972

42 Zhu Y, Xu C, Zhang N, et al. Polycationic synergistic antibacterial agents with multiple functional components for efficient anti-infective therapy. Adv Funct Mater, 2018, 28: 1706709

43 Chen Z, Ji H, Liu C, et al. A multinuclear metal complex based DNasemimetic artificial enzyme: Matrix cleavage for combating bacterial biofilms. Angew Chem Int Ed, 2016, 55: 10732-10736

44 Niu J, Sun Y, Wang F, et al. Photomodulated nanozyme used for a Gram-selective antimicrobial. Chem Mater, 2018, 30: 7027-7033

45 Sun Y, Zhao C, Niu J, et al. Colorimetric band-aids for point-of-care sensing and treating bacterial infection. ACS Cent Sci, 2020, 6: 207-212

46 Sayed SM, Xu KF, Jia HR, et al. Naphthalimide-based multifunctional AIEgens: Selective, fast, and wash-free fluorescence tracking and identification of Gram-positive bacteria. Anal Chim Acta, 2021, 1146: 41-52

47 Yang J, Gao G, Zhang X, et al. One-step synthesis of carbon dots with bacterial contact-enhanced fluorescence emission: Fast Gram-type identification and selective Gram-positive bacterial inactivation. Carbon, 2019, 146: 827-839

48 Zhang X, Chen X, Yang J, et al. Quaternized silicon nanoparticles with polarity-sensitive fluorescence for selectively imaging and killing Grampositive bacteria. Adv Funct Mater, 2016, 26: 5958-5970

Acknowledgements This work was supported by the National Natural Science Foundation of China (52003222 and 21875189), Ningbo Natural Science Foundation (202003N4064), the Natural Science Foundation of Chongqing (cstc2020jcyj-msxmX0752), the Joint Research Funds of Department of Science \& Technology of Shaanxi Province and Northwestern Polytechnical University (2020GXLH-Z-013), and the Fundamental Research Funds for the Central Universities.

Author contributions Feng T, Li P, and Huang W conceived the idea and supervised the study; Feng T, Ye X, and Lu H performed the experiments and wrote the manuscript; Nie C, Zhang J, Yu L, and Jin $\mathrm{H}$ participated in the in vivo anti-infective assay. All authors contributed to the general discussion. 
Conflict of interest The authors declare that they have no conflict of interest.

Supplementary information Experimental details and supporting data are available in the online version of the paper.

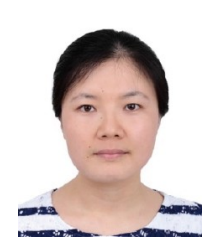

Tao Feng received her BSc and MSc degrees from Jilin University in 2010 and 2013, and $\mathrm{PhD}$ degree from Nanyang Technological University in 2018. She is currently an associate professor at Northwestern Polytechnical University. Her research focuses on the design of fluorescent probes for selective discrimination and accurate killing of bacteria.

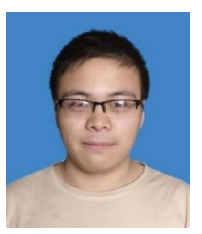

Hui Lu received his BSc degree from Anhui University of Technology in 2019. Currently, he is a graduate student at the Frontiers Science Center for Flexible Electronics, Northwestern Polytechnical University. His research interest is developing new phototheranostic agents to selectively inactivate bacteria.

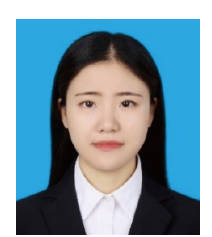

Xiaoting Ye received her BSc degree from Nanjing Tech University in 2018 and MSc degree from the Frontiers Science Center for Flexible Electronics, Northwestern Polytechnical University in 2021. Her research focuses on preparing novel photothermal materials for specific killing of bacteria.

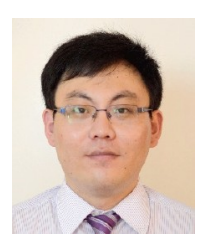

Peng Li received his BSc from Tianjin University in 2006 and $\mathrm{PhD}$ degree from Nanyang Technological University in 2013. He has been a professor of the Frontiers Science Center for Flexible Electronics at Northwestern Polytechnical University since 2018. His research interest is developing innovative antibacterial materials and strategies for infectious diseases.

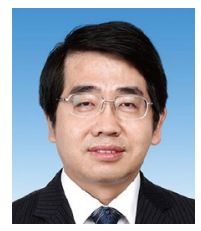

Wei Huang received his BSc degree from Peking University in 1983, followed by MSc and $\mathrm{PhD}$ degrees from the same university. Then he did his postdoctoral research at the National University of Singapore, where he participated in the foundation of the Institute of Materials Research and Engineering in 1995. In 2011, he was elected as academician of the Chinese Academy of Sciences. Now, he is the chief scientist of the Frontiers Science Center for Flexible Electronics at Northwestern Polytechnical University. His research interests include polymer sciences, bioelectronics, nanoelectronics, and organic/plastic/flex-

ible electronics.

\section{代谢标记策略用于体外和体内革兰氏阳性菌的选择 性杀灭}

冯涛 ${ }^{1+*}$, 陆辉 ${ }^{1 \dagger}$, 叶晓婷 ${ }^{1 \dagger}$, 聂超凡 ${ }^{1}$, 张建红 ${ }^{1}$, 余骆峰 ${ }^{1}$, 靳吴宇 ${ }^{1}$, 李鹏 ${ }^{*}$, 黄维 $1,2,3$

摘要 细菌感染, 尤其是最常见的革兰氏阳性菌引起的感染, 严重威胁 着人类的生命健康. 抗生素是治疗细菌感染的首选药物, 但是它的大量 使用造成了耐药性细菌的快速滋生和曼延. 近年来, 光热抗菌疗法作为 一种不易引起细菌耐药性的技术受到了广泛的关注. 然而, 非特异性的 细菌杀伤和对邻近哺乳动物细胞的毒副作用严重限制了光热抗菌疗法 的实际应用. 本文开发了一种代谢标记策略用于革兰氏阳性菌的选择 性光热杀灭. 通过二苯并环辛炔(DBCO)和IR-780硾化物的共价连接, 制备了生物正交的光热试剂IR780-DBCO. 革兰氏阳性菌或阴性菌经过 3 - 叠氮基- $D$-丙氨酸预处理, 利用无铜点击化学反应实现了IR780DBCO在金黄色葡萄球菌和耐万古霉素肠球菌等革兰氏阳性菌表面的 选择性标记. 在 $15 \mathrm{~min}$ 的近红外光照射下, 接近 $100 \%$ 的革兰氏阳性菌被 有效地杀灭. 此外, 该方法在小鼠皮肤伤口感染模型中也能够显著地抑 制耐万古霉素肠球菌引起的感染. 我们提出的基于代谢标记的特异性 光热杀灭革兰氏阳性菌的策略将会促进预临床研究中精准抗菌试剂的 研发. 